\title{
Treatment of Intracranial Aneurysms with Flow Re-direction Endoluminal Device - A Single Centre Experience with Short-term Follow-up Results
}

\author{
Neeraj Ramesh Mahboobani, MD², Wing Ho Chong, MD', Samuel Siu Kei Lam, MD³, \\ Jimmy Chi Wai Siu, MD', Chong Boon Tan, MD', Yiu Chung Wong, MD'
}

Purpose: A flow diverter (FD) is an effective treatment option for intracranial aneurysms. The Flow Redirection Endoluminal Device (FRED) is a relatively new flow diverter with a unique dual-layer design. We report our experience and short-term results with the FRED.

Materials and Methods: We did a retrospective review of all consecutive cases in which the FRED was used to treat intracranial aneurysms at a single institution from March 2014 till December 2015. Clinical parameters, aneurysm characteristics, technical results and short-term outcomes were reviewed.

Results: Eleven intracranial aneurysms were treated with the FRED in 11 patients. The technical device deployment success rate was $100 \%$. Immediate reduction in intra-aneurysmal flow after deployment was noted in 10 cases. The aneurysm occlusion rate at 6 months was $75 \%$. There was 1 complication of in-stent thrombosis immediately after deployment. There was no side branch occlusion, delayed aneurysm rupture, stroke, or intraparenchymal haemorrhage. There was no neurological deficit, morbidity, or mortality.

Conclusion: The FRED is a new FD. It has shown to be safe and effective in our series. The unique duallayer design of the device renders it to have technical advantages over other FDs. The 6-month aneurysm occlusion rate and complication profile of FRED are similar to other FDs.

Key Words : Intracranial aneurysm; stents; flow diversion; flow diverters; FRED;

Flow Re-direction Endoluminal Device

'Department of Radiology, Tuen Mun Hospital, Hong Kong

2Department of Radiology, Princess Margaret Hospital, Hong Kong

${ }^{3}$ Department of Neurosurgery, Tuen Mun Hospital, Hong Kong

Received October 15, 2016;

accepted after revision January 2, 2017.

Correspondence to: Neeraj Ramesh Mahboobani, MD, Department of Radiology, Princess Margaret Hospital, Lai Chi Kok, Kowloon, Hong Kong. Tel. 852.97453454 Fax. 852.29903276

E-mail: neeraj.mahboobani@gmail.com

This is an Open Access article distributed under the terms of the Creative Commons Attribution Non-Commercial License (http://creativecommons.org/licenses/by-nc/3.0) which permits unrestricted non-commercial use, distribution, and reproduction in any medium, provided the original work is properly cited. 
A flow diverter (FD) is an effective treatment option for intracranial aneurysms [1-3]. FDs are stent-like devices that are deployed in the parent artery. They alter flow dynamics at the interface between the parent artery and the aneurysm. This alteration results in stasis of intra-aneurysmal flow, aneurysm thrombosis as well as endoluminal reconstruction of the parent artery by endothelialization along the device scaffold.

There are a number of FDs that are available for clinical use. These include but are not limited to the Pipeline Embolization Device (PED; Covidien, Irvine, California, USA), SILK flow diverter (SILK; Balt Extrusion, Montmorency, France) and Surpass (Surpass; Stryker Neurovascular, Fremont, California, USA).

The Flow Re-direction Endoluminal Device (FRED; MicroVention, Tustin, California, USA) is a relatively new FD. There are limited reports on its use and clinical outcomes [4-7]. We report our experience and short-term results with the FRED.

\section{MATERIALS AND METHODS}

This study was approved by the institutional review board (IRB) at the participating institution. We conducted a retrospective review of all consecutive cases in which the FRED was used to treat intracranial aneurysms at a single institution between March 2014 and December 2015. Patient demographics, clinical presentation, aneurysm characteristics, technical aspects, pre-treatment, immediate post-treatment and 6month post-treatment digital subtraction angiograms (DSA), and clinical outcomes were reviewed.

\section{Institutional treatment indications}

Our institutional treatment indications for use of the FRED were as follows: 1) wide-neck saccular aneurysms with dome:neck ratio $<2$ or neck $>4 \mathrm{~mm}$; 2) Large ( $>10 \mathrm{~mm})$ aneurysms or aneurysms with mass effect; 3 ) fusiform aneurysms; 4) dissecting aneurysms;

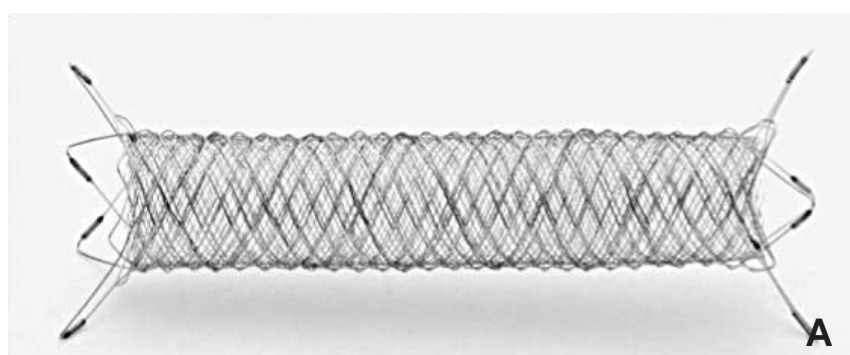

and 5) recanalization of previously coiled aneurysms.

\section{Device}

Second generation FREDs were used. The FRED is a self-expandable, closed-cell stent. It is composed of braided nitinol wires, and has a unique dual-layer design which is comprised of a 16 -wire high porosity outer stent forming the total length of the stent and a 48-wire low porosity inner stent in the midsection of the outer stent forming the working length of the stent, with the peripheral 2-4 $\mathrm{mm}$ at each end of the stent formed only by the high porosity outer stent (Fig. 1). The flow diversion effect is achieved only along the working length of the stent.

The inner stent is attached to the outer stent by a double helix of radiopaque tantalum, which runs along the entire length of the inner stent. Each end of the outer stent is flared and marked with 4 radiopaque tips. Both the working length and total length can be delineated under fluoroscopy as marked by the double helix interwoven tantalum wire and the 4 proximal / distal radiopaque tips respectively.

The FRED is attached to a delivery microwire, which has a radiopaque distal tip and a proximal marker. It is tracked and deployed by a 0.027 inch Headway 27 microcatheter (MicroVention). The stent is deployed once the Headway 27 microcatheter is fully withdrawn over the device on the delivery microwire.

\section{Endovascular Procedure}

All procedures were performed with the patient under general anaesthesia and with a unilateral femoral approach. DSA with 3D volumetric reconstruction was done for each case using a biplane angiographic system (Axiom Artis, Siemens, Erlangen, Germany) to delineate the anatomy of the parent artery and measure its diameter proximal and distal to the aneurysm.

The FRED diameter was selected based on the maximum diameter of the segment of the parent artery in which the device was being deployed into. The

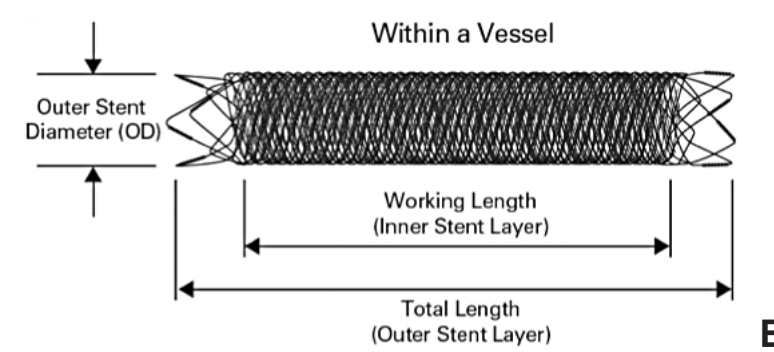

Fig. 1. The FRED device. Profile view (A) and illustration (B) of the FRED showing dual-layer design. Reproduced with permission from MicroVention, Inc. 


\section{Treatment of Aneurysms with Flow Re-direction Endoluminal Device}

working length was selected in order to be sufficient to cover the neck of the aneurysm or the length of the fusiform or dissecting aneurysm, with a margin of at least $3 \mathrm{~mm}$ at both the proximal and distal ends.

The FRED was delivered tri-axially using a $6 \mathrm{Fr}$ Neuron 070 guiding catheter (Penumbra, Alameda, California, USA) with an intermediate 5Fr Sofia catheter (MicroVention) and Headway 27 microcatheter. A 0.014 inch Traxcess microguidewire (MicroVention) was used in all cases.

The Headway 27 microcatheter was first positioned distal to the aneurysm under roadmap guidance. The FRED was then inserted and then partially deployed until there was flaring of the 4 distal radiopaque tip markers. The entire construct of the partially deployed FRED and the microcatheter were then withdrawn proximally to the desired position. The FRED was then fully deployed by unsheathing the Headway 27 microcatheter while maintaining antegrade tension on the FRED delivery microwire under constant biplane fluoroscopy.

If an incomplete FRED opening was noted, in-stent angioplasty was performed using a Scepter C compliant balloon (MicroVention) followed by subsequent DSA and cone-beam CT (DynaCT) to evaluate FRED apposition to parent artery wall.

\section{Anti-platelet and anti-coagulation protocol}

All patients received dual-antiplatelet (DAT) loading with aspirin $320 \mathrm{mg}$ and clopidogrel $300 \mathrm{mg} 1$ day before the procedure. This was followed by maintenance DAT with aspirin $80 \mathrm{mg}$ and clopidogrel $75 \mathrm{mg}$ daily for 3 months and subsequently followed by lifelong single antiplatelet with aspirin $80 \mathrm{mg}$ daily.

There was no routine testing of individual aspirin and clopidogrel response from March 2014 - August 2015. From September 2015 onwards, routine testing of aspirin and clopidogrel response was implemented using the VerifyNow (Accumetrics Inc., San Diego, California, USA) system. Clopidogrel hyporesponders were given ticagrelor (loading dose $180 \mathrm{mg}$; maintenance dose $90 \mathrm{mg}$ bd together with aspirin $80 \mathrm{mg}$ daily for 3 months).

All patients were anticoagulated with heparin 3,000IU after diagnostic angiograms and were given an additional bolus of 1,000IU every hour throughout the procedure. Activated clotting time was not routinely measured. There was no reversal of anticoagulation at the end of the procedure.

\section{Angiographic evaluation}

Pre-treatment, immediate post-treatment, and 6month post-treatment DSA were evaluated for degree of filling and stasis of flow within the aneurysm using the O'Kelly-Marotta (OKM) grading scale [8]. This scale has been reported to have good intra- and interobserver agreement [9]. Each aneurysm is graded with a letter, which represents the degree of filling (A, total filling; B, subtotal filling; C, entry remnant; D, no filling). This grade is followed by a number, which represents the degree of stasis (stasis into 1, arterial phase; 2 , capillary phase; 3 , venous phase) in grades AC.

\section{Clinical evaluation}

Neurological examination including modified Rankin scale ( $\mathrm{mRS}$ ) was done at baseline before the procedure, immediately after the procedure, at discharge and at interval outpatient follow-up.

\section{RESULTS}

\section{Demographics and clinical presentation}

Eleven intracranial aneurysms were treated with the FRED in 11 patients ( 9 females; mean age 51.8 years, range 38-62 years). Six patients were asymptomatic, 2 patients had neurological complaints, 2 had previously coiled aneurysms with recanalization, and 1 presented with acute subarachnoid hemorrhage after rupture of a large wide-neck paraophthalmic aneurysm. Demographics and clinical presentations are detailed together with aneurysm characteristics in Table 1.

\section{Aneurysm characteristics}

Eight aneurysms were located in the anterior circulation in the distal internal carotid artery (ICA). Three were in the posterior circulation in the vertebral artery. There were 7 wide-neck saccular aneurysms, 2 dissecting aneurysms, and 2 previously coiled saccular aneurysms with recanalization (Table 1).

\section{Technical results}

In all 11 cases, only the FRED was used without any additional embolic materials such as coils. In 10 out of the 11 cases, a single FRED was deployed. Two FREDs were deployed in an overlapping manner in the case of the large ruptured aneurysm (case 7). The technical device deployment success rate was $100 \%$.

Complete apposition of the FRED to the arterial wall after deployment was seen in 10 cases. Near complete $(>90 \%)$ FRED apposition to the parent artery was 
Neeraj Ramesh Mahboobani, et al.

Table 1. Patient Demographics, Clinical Presentation, Aneurysm Characteristics, FRED Device Utilized, Aneurysm Filling, Complications and Modified Rankin Scale

\begin{tabular}{|c|c|c|c|c|c|c|c|c|c|c|c|c|c|c|}
\hline Case & $\begin{array}{c}\text { Sex } \\
\text { (years) }\end{array}$ & Age & Presentation & Location & Type & $\begin{array}{l}\text { Size } \\
(\mathrm{mm})\end{array}$ & $\begin{array}{l}\text { Dome / } \\
\text { neck } \\
(\mathrm{mm})\end{array}$ & $\begin{array}{l}\text { Parent } \\
\text { artery p/d } \\
(\mathrm{mm})\end{array}$ & $\begin{array}{l}\text { FRED } \\
\text { size } \\
(\mathrm{mm})\end{array}$ & $\begin{array}{l}\text { OKM } \\
\text { grade } \\
\text { before/after } \\
\text { deployment }\end{array}$ & $\begin{array}{l}\text { OKM } \\
\text { grade at } \\
\text { 6-months }\end{array}$ & Complication & $\begin{array}{l}\text { Baseline / } \\
\text { Pre-morbid } \\
\text { mRS }\end{array}$ & $\begin{array}{l}\text { Latest } \\
\text { follow-up } \\
\text { mRS }\end{array}$ \\
\hline 1 & $F$ & 54 & Incidental & LVA & Dis & $5 \times 7$ & Dis & $3.5 / 3.3$ & $3.5 \times 22$ & $\mathrm{~A} 1 / \mathrm{A} 3$ & D & - & 0 & 0 \\
\hline 2 & $\mathrm{~F}$ & 60 & Diplopia & L Cav ICA & $S$ & $26 \times 21$ & $26 / 16$ & $5.1 / 4.8$ & $5.0 \times 26$ & $\mathrm{~A} 1 / \mathrm{A} 2$ & B2 & - & 0 & 0 \\
\hline 3 & $\mathrm{~F}$ & 57 & Incidental & R PO ICA & $S$ & $3 \times 4$ & $3 / 2$ & $4.6 / 4.2$ & $4.5 \times 34$ & $\mathrm{~A} 1 / \mathrm{A} 2$ & A3 & - & 0 & 0 \\
\hline 4 & $\mathrm{~F}$ & 42 & Headache & R VA & Dis & $8 \times 9$ & Dis & $3.5 / 3.3$ & $3.5 \times 22$ & $\mathrm{~A} 1 / \mathrm{A} 3$ & D & - & 0 & 0 \\
\hline 5 & $\mathrm{~F}$ & 51 & Recanalization & R PO ICA & $S$ & $3 \times 5$ & $3 / 3$ & $4.0 / 3.8$ & $4.0 \times 23$ & $\mathrm{~A} 1 / \mathrm{A} 1$ & D & - & 0 & 0 \\
\hline 6 & $\mathrm{~F}$ & 62 & Incidental & LPO ICA & S & $3 \times 4$ & $3 / 3$ & $4.5 / 4.2$ & $4.5 \times 25$ & $\mathrm{~A} 1 / \mathrm{A} 2$ & D & - & 0 & 0 \\
\hline 7 & M & 38 & Ruptured & LPO ICA & $S$ & $10 \times 13$ & $10 / 7$ & $4.5 / 4.5$ & $\begin{array}{l}4.5 \times 25 \\
4.5 \times 25\end{array}$ & $\begin{array}{l}\mathrm{A} 1 / \mathrm{A} 1 \\
\mathrm{~A} 1 / \mathrm{A} 3\end{array}$ & D & - & 0 & 0 \\
\hline 8 & $F$ & 47 & Incidental & LPO ICA & $S$ & $4 \times 4$ & $4 / 3$ & $4.0 / 3.5$ & $4.0 \times 23$ & $\mathrm{~A} 1 / \mathrm{A} 2$ & D & - & 0 & 0 \\
\hline 9 & $\mathrm{M}$ & 53 & Incidental & LPO ICA & $S$ & $23 \times 21$ & $23 / 6$ & $4.0 / 3.8$ & $4.0 \times 32$ & $\mathrm{~A} 1 / \mathrm{C} 3$ & NA & $\begin{array}{l}\text { FRED } \\
\text { thrombosis }\end{array}$ & 0 & 0 \\
\hline 10 & $\mathrm{~F}$ & 60 & Incidental & R PO ICA & S & $5 \times 5$ & $5 / 4$ & $3.9 / 4.0$ & $4.0 \times 23$ & $\mathrm{~A} 1 / \mathrm{A} 2$ & NA & - & 0 & 0 \\
\hline 11 & $\mathrm{~F}$ & 46 & Recanalization & R VA & S & $6 \times 5$ & $6 / 5$ & $3.3 / 3.5$ & $3.5 \times 22$ & $\mathrm{~A} 1 / \mathrm{A} 3$ & NA & - & 0 & 0 \\
\hline
\end{tabular}

Cav, Cavernous; Dis, dissecting; d, distal; ICA, internal carotid artery; L, left; mRS, modified Rankin scale; NA, not available; PO, paraophthalmic; p, proximal; R, right; S, saccular; VA, vertebral artery

noted after deployment in 1 case and this was augmented with Scepter C compliant balloon.

\section{Procedural complications}

There was 1 procedural complication of acute complete in-stent thrombosis shortly after FRED deployment in the distal ICA (Fig. 2). Minimal recanalization was achieved with $15 \mathrm{mg}$ intra-arterial abciximab (ReoPro, Janssen Biologics BV, Leiden, Netherlands). Adequate collateral circulation through the Circle of Willis and leptomeningeal anastomoses was seen on vertebral and contralateral ICA DSA. There was no immediate motor or sensory deficit.

A follow-up CT angiogram 2 weeks after the procedure showed occlusion of the ipsilateral ICA, FRED and the large ICA aneurysm (Fig. 2). The ipsilateral anterior and middle cerebral arteries were supplied by the vertebrobasilar circulation and contralateral ICA via the Circle of Willis. There was no delayed neurological deficit or change from baseline mRS.

Pre-treatment, immediate post-treatment and 6-month post-treatment angiographic results

All aneurysms showed complete filling without stasis
(OKM grade A1) before treatment. Immediate reduction of intra-aneurysmal flow resulting in a change of OKM grade was noted on control angiogram immediately after FRED deployment in 10 aneurysms. 6-month follow-up DSA available in 8 patients showed complete occlusion (OKM grade D) in 6 out of 8 $(75 \%)$ of aneurysms. The remaining 2 aneurysms were OKM grade B2 and grade A3 at 6 months. There was no side branch occlusion, FRED foreshortening, kinking or migration on 6-month follow-up.

\section{Clinical evaluation}

There was no new neurological deficit or change from baseline $\mathrm{mRS}$ in all cases.

\section{DISCUSSION}

The ultimate aim of treatment with FDs is to achieve complete aneurysm occlusion while maintaining patency of the parent artery, its side branches and perforators. Thrombosis of aneurysms is often a gradual process, which ensues over a period of weeks to months, after FD deployment [2]. The 6-month aneurysm occlusion rate of $75 \%$ in our series is comparable to the 4-6 month aneurysm occlusion rate 
of $80 \%$ in the series by Kocer et al [6], and the 6-month aneurysm occlusion rate of $73 \%$ in the series by Mohlenbruch et al [5]. This in turn is similar to the 6month aneurysm occlusion rate of $76 \%$ with the PED and SILK reported in a meta-analysis reviewing 29 studies [1].

In our series, 2 aneurysms remained patent at the 6month follow-up. These aneurysms showed further
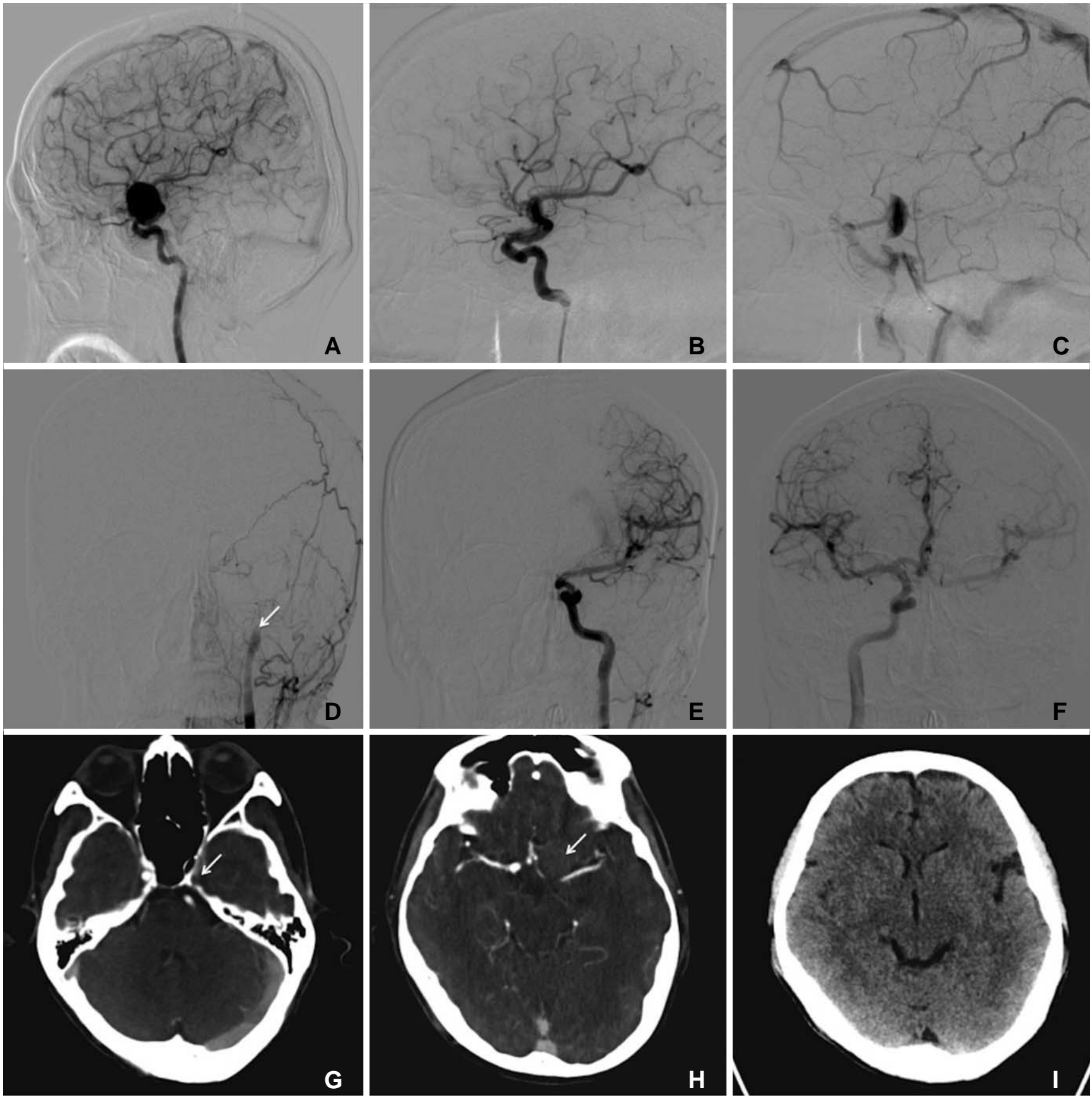

Fig. 2. A 53-year-old man with large left paraophthalmic internal carotid artery (ICA) aneurysm (case 9). (A) Lateral view of left ICA angiogram showing large paraophthalmic aneurysm. (B, C) Immediate post-deployment lateral view of left ICA angiogram at arterial (B) and venous $(\mathbf{C})$ phases with layering of contrast seen on venous (C) phase. (D) Post-deployment anteroposterior (AP) view of left common carotid artery (CCA) angiogram showing no opacification of left ICA beyond the vertical part of petrous segment (arrow). (E) AP view of left ICA angiogram after intra-arterial thrombolysis with abciximab showing partial recanalization of left intracranial ICA. (F) AP view of right ICA angiogram showing crossflow to left anterior (ACA) and middle (MCA) cerebral arteries via anterior communicating artery. (G) CT angiogram of Circle of Willis acquired 2 weeks after FRED deployment showing occluded left ICA (arrow). (H) CT angiogram of Circle of Willis acquired 2 weeks after FRED deployment showing thrombosed aneurysm (arrow) and patent left ACA and MCA. (I) Non-contrast CT brain done prior to CT angiogram of Circle of Willis with image at level of lentiform nuclei - no infarct is seen. 


\section{Neeraj Ramesh Mahboobani, et al.}

alterations in intra-aneurysmal flow with an increase in the degree of stasis or decrease in degree of aneurysmal filling at the 6-month follow-up when compared with immediate post-deployment angiograms. Persistent filling of these aneurysms can be related to less than ideal coverage of the aneurysmal neck by the working length of the device. Aneurysm occlusion rates with the FRED have been shown to continue to increase between 4-6 and 7-12 months post-deployment [6]. Thus, our management plan for these 2 patients is surveillance of aneurysmal filling with DSA every 6 months up till a total of 18 months post-deployment. Deployment of an additional FRED will be considered if persistent intra-aneurysmal flow is noted at 18 months.

Thrombosis of FD is a recognized complication and it can occur as early as immediately after FD deployment, in the peri-procedural period or even months after FD deployment. Such complications have also been reported with the FRED. Mohlenbruch et al reported 2 cases of in-stent thrombosis [5]. One was noted minutes after FRED deployment and was reversible without clinical sequelae. The other was related to discontinuation of antiplatelet 2 months after FRED deployment, and this was complicated by embolic infarcts. Poncyljusz et al reported 1 case of delayed asymptomatic complete FRED thrombosis on follow-up DSA at 3 months [7].

We had one case of asymptomatic in-stent thrombosis immediately after FRED deployment in paraophthalmic ICA. Intra-arterial chemical thrombolysis transiently mitigated the degree of parent artery thrombosis. The ICA was occluded on subsequent imaging 2 weeks after the procedure. There was adequate collateral circulation via Circle of Willis. Clinical evaluation subsequently revealed that the patient was a clopidogrel hyporesponder, which we attribute to be the reason for the complication.

The percentage of clopidogrel hyporesponders in studies evaluating clopidogrel responder status with complications following PED procedures ranges from 4 $-52.7 \%$ [10-12]. Clopidogrel is a prodrug that is converted into its active form in the liver by the cytochrome P450 system. The active metabolite of clopidogrel causes irreversible inhibition of the P2Y12 receptor, which plays an important role in platelet activation and aggregation. Clopidogrel response can vary in individuals as a result of intrinsic factors such as P450 enzyme gene polymorphism, variable intestinal absorption, or extrinsic factors such as drug-drug interactions.
Clopidogrel response can be measured using the VerifyNow system, which is a point-of-care platelet function test. This system reports results in terms of P2Y12 reaction units (PRU). Increased clopidogrel dose, or alternative $\mathrm{P} 2 \mathrm{Y} 12$ receptor inhibitors such as prasugrel or ticagrelor, can be considered to attain adequate $\mathrm{P} 2 \mathrm{Y} 12$ receptor inhibition in clopidogrel hyporesponders.

Measurement of P2Y12 inhibition before FD placement was not routine in our institution. Subsequent to identification of the clopidogrel hyporesponder, our institutional protocol for DAT was modified to include evaluation of aspirin and P2Y12 response in patients prior to FD placement using the VerifyNow system. We subsequently identified another clopidogrel hyporesponder in our series. Adequate P2Y12 inhibition was attained in this patient with ticagrelor.

Tan et al reported a trend of increased risk of symptomatic thromboembolic events following PED procedures in patients with $\mathrm{P} 2 \mathrm{Y} 12$ receptor underinhibition [11]. Delgado Almandoz et al reported a statistically significant association between P2Y12 receptor under-inhibition status and 30-day periprocedural thromboembolic complications following PED procedures in their cohort [10].

Though the body of evidence to support personalized antiplatelet regimens in clopidogrel hyporesponders who undergo PED deployment is limited, it is plausible that P2Y12 under-inhibition may place these patients at risk of developing peri-operative and delayed thromboembolic complications. We hypothesize this applies to the FRED as well given the case of FRED thrombosis in our series. The case of complete in-stent thrombosis following termination of P2Y12 inhibitor 2 months after deployment in the series by Mohlenbruch et al further substantiates our hypothesis [5].

No delayed in-stent thrombosis, stent migration, or changes in stent morphology such as inward crimping of stent ends, foreshortening or kinking was seen in our series on DSA at 6 months. Mohlenbruch et al also reported similar findings on DSA at 3 months [5]. Second generation FREDs were used in both series. In the earlier series by Kocer et al, inward crimping of FRED ends was noted in 4 cases on follow-up DSA [6]. The authors highlighted that 1st generation FREDs were used in these 4 cases. Poncyljusz et al reported a case of asymptomatic complete FRED thrombosis on follow-up DSA at 3 months, which they attributed to inward crimping of the distal end of the FRED [7]. There is however no information on the generation of 


\section{Treatment of Aneurysms with Flow Re-direction Endoluminal Device}

the FRED used in that case.

Side branches are often covered when FD are deployed. Although the construct of FD is designed to maintain patency of side branches even when they are covered, side branch occlusions have been reported [13, 14]. All covered side branches in our case series remained patent on follow-up DSA at 6 months (Figs. $3,4)$, including the ophthalmic artery in the case of the large ruptured paraophthalmic aneurysm (case 7), which was covered by the working length of 2 overlapping FREDs. The wide-neck aneurysm in this case involved more than half the circumference of the parent ICA, and conventional treatment of coiling in the acute phase of rupture was rendered to carry a significant risk of coil herniation into the parent ICA. Neurosurgical treatment was rendered to be technically difficult given the size, configuration and location of the aneurysm. Thus it was decided to treat the aneurysm with a FD. There was no reduction in intra- aneurysmal flow after deployment of a single FRED. An additional FRED was then deployed in an overlapping manner after re-crossing the aneurysm and the 1st device. Significant stasis was seen within the aneurysm after deployment of the 2 nd device (Fig. 3). The aneurysm was occluded on follow-up DSA at 6 months with no angiographic or clinical complications (Fig. 3). This case in our series seems to be the only reported case in literature thus far on the use of overlapping FREDs.

With regard to the technical aspects of the FRED, we experienced smooth navigation and deployment when compared to our previous experience with a PED. We attribute this technical advantage to the device's novel dual-layer design, which has a 16-wire high porosity outer stent in contact with its delivery microcatheter. This design is the likely explanation for the reduced friction between the device and its delivery microcatheter, in comparison to our experience with the PED,
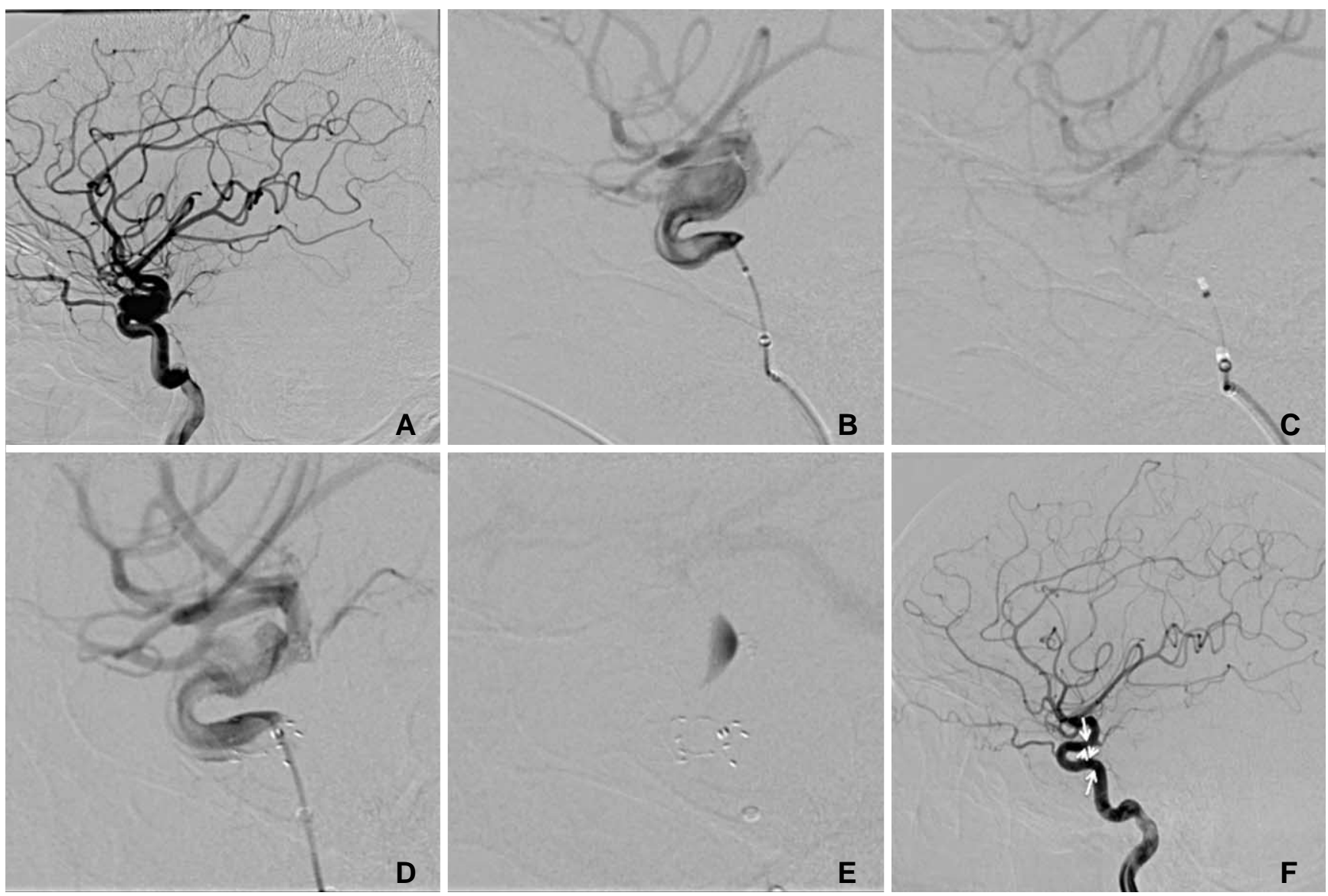

Fig. 3. A 38-year-old man with large ruptured left paraophthalmic internal carotid artery (ICA) aneurysm (case 7). (A) Lateral view of left ICA angiogram showing large paraophthalmic aneurysm. (B, C) Angiogram after deployment of the first FRED showing opacification of aneurysm (B) with contrast washout (C) in sync with ICA indicative of rapid flow within aneurysm. (D, E) Angiogram after deployment of a second FRED in an overlapping manner showing opacification of aneurysm (D) with contrast stasis and layering (E). (F) 6-month followup angiogram with illustrated working length (arrowheads) and total length (arrows) of overlapping FREDs. The aneurysm is occluded. Left ophthalmic artery is patent. 

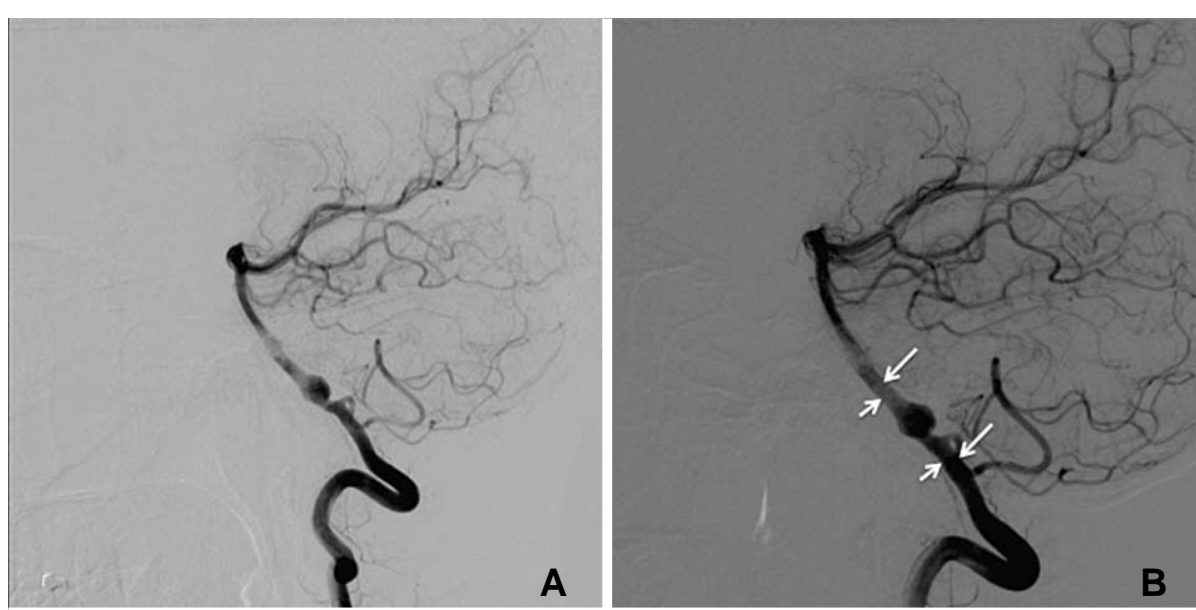

Fig. 4. A 42-year-old woman with dissecting fusiform aneurysm in V4 segment of right vertebral artery (VA; case 4). (A) Lateral view of right VA angiogram showing the aneurysm shortly distal to origin of right posterior inferior cerebellar artery (PICA). (B) Arterial phase of immediate post-FRED deployment angiogram with illustrated working length (short arrows) and total length (long arrows) of the FRED. (C) Venous phase of immediate postdeployment angiogram showing layering of contrast within the aneurysm. (D) 6month follow-up angiogram showing occluded aneurysm and patent right
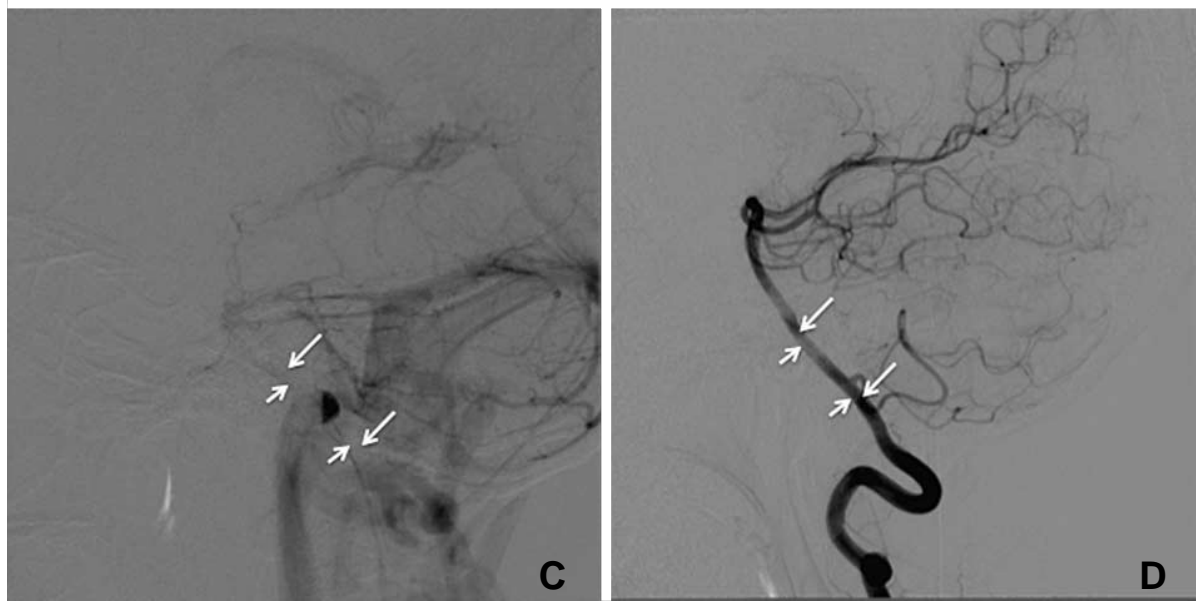
PICA.

which is single-layered with 48 wires.

Adequate apposition of flow diverters to the vessel wall is important in order to avoid continuous filling of the aneurysm. The device was seen to open and appose well to the parent artery wall in all cases in our series. In particular, the device was seen to maintain its cylindrical shape well with no kinking or bending when used around tortuous carotid siphons. We believe this is also related to the device's dual layer design with the outer and inner stents providing additive radial forces during deployment and opening.

The proximal and distal 2-4 $\mathrm{mm}$ of the device consists only of the high porosity outer stent with no flow diversion effect, and we found this facilitates additional anchorage of the device in the parent artery without risk of perforator or side-branch occlusion. Retrievability of the device after partial deployment is an essential feature especially when deploying the device in tortuous vessels where re-positioning may be necessary. Although the vendor informs that the device can be re-sheathed provided that $50 \%$ or less of its total length has been deployed, previous case series have described that the device is retrievable as long as $80 \%$ or less of its length has been deployed [5-7].

The FRED was the sole treatment device we used in our series. We used an average of 1.1 FREDs to treat each aneurysm (12 FRED for 11 aneurysms). One FRED was used in each patient in other reported case series, and in some cases, multiple aneurysms were treated with a single device [4-7]. These numbers contrast with the average number of 2 PEDs used per aneurysm (range 1.0-3.2) reported in a systematic review by Leung et al [15]. We postulate that this difference is, in part at least, related to two factors of the device itself. First, long working lengths of the FRED (range 7-56 $\mathrm{mm}$ ) are available, thereby likely reducing the number of devices needed in cases of multiple aneurysms. Second, the FRED can be resheathed and repositioned, thereby enabling more accurate deployment in tortuous parent arteries. We also postulate that a smaller average number of FREDs needed to treat each aneurysm may result in shorter procedural times when compared with the PED, and this may be worth investigating further.

We acknowledge that the retrospective nature, a limited number of 11 aneurysms from a single center, 
and a short follow-up period of 6 months are limitations of this study. Nonetheless, our objective was to add to the body of literature our experience and early outcomes with the FRED, which corroborates with the observations and outcomes reported in the limited number of studies on the FRED thus far. Given that the 6-month aneurysm occlusion rate is similar to other FDs, the number of reported complications small, and its novel dual-layer design which offers technical advantages over previous FD, the FRED seems to be a promising FD for the treatment of intracranial aneurysms which warrants larger multicentre trials.

\section{CONCLUSION}

The FRED is a new FD. It was noted to be safe and effective in the treatment of intracranial aneurysms in our series. It has a novel dual-layer design, which offers technical advantages over other FDs, whilst maintaining a comparable 6-month aneurysm occlusion rate and complication profile with other FDs. This renders the device promising and we believe large-scale multicentre studies of the device are warranted to further establish its clinical use.

\section{References}

1. Brinjikji W, Murad MH, Lanzino G, Cloft HJ, Kallmes DF. Endovascular treatment of intracranial aneurysms with flow diverters: a meta-analysis. Stroke 2013;44:442-447

2. Alderazi YJ, Shastri D, Kass-Hout T, Prestigiacomo CJ, Gandhi CD. Flow diverters for intracranial aneurysms. Stroke Res Treat 2014;2014:415-653

3. Zanaty M, Chalouhi N, Tjoumakaris SI, Rosenwasser RH, Gonzalez LF, Jabbour P. Flow-diversion panacea or poison?. Front Neurol 2014;5:21

4. Diaz O, Gist TL, Manjarez G,Orozco F, Almeida R. Treatment of 14 intracranial aneurysms with the FRED system. J Neurointerv Surg 2014;6:614-617

5. Möhlenbruch MA, Herweh C, Jestaedt L, Stampfl S,
Schonenberger S, Ringleb PA, et al. The FRED flow-diverter stent for intracranial aneurysms: clinical study to assess safety and efficacy. AJNR Am J Neuroradiol 2015;36:1155-1161

6. Kocer N, Islak C, Kizilkilic O, Kocak B, Saglam M, Tureci E. Flow Re-direction Endoluminal Device in treatment of cerebral aneurysms: initial experience with short-term follow-up results. $J$ Neurosurg 2014;120:1158-1171

7. Poncyljusz W, Sagan L, Safranow K, Rac M. Initial experience with implantation of novel dual layer flow-diverter device FRED. Wideochir Inne Tech Maloinwazyjne 2013;8:258-264

8. O'Kelly CJ, Krings T, Fiorella D, Marotta TR. A novel grading scale for the angiographic assessment of intracranial aneurysms treated using flow diverting stents. Interv Neuroradiol 2010;16:133-137

9. Joshi MD, O’Kelly CJ, Krings T, Fiorella D, Marotta TR. Observer variability of an angiographic grading scale used for the assessment of intracranial aneurysms treated with flow-diverting stents. AJNR Am J Neuroradiol 2013;34:1589-1592

10. Delgado Almandoz JE, Crandall BM, Scholz JM, Fease JL, Anderson RE, Kadkhodayan Y, et al. Pre-procedure P2Y12 reaction units value predicts perioperative thromboembolic and hemorrhagic complications in patients with cerebral aneurysms treated with the Pipeline Embolization Device. J Neurointerv Surg 2013;5:iii3-10

11. Tan LA, Keigher KM, Munich SA, Moftakhar R, Lopes DK. Thromboembolic complications with Pipeline Embolization Device placement: impact of procedure time, number of stents and pre-procedure $\mathrm{P} 2 \mathrm{Y} 12$ reaction unit (PRU) value. J Neurointerv Surg 2015;7:217-221

12. Heller RS, Dandamudi V, Lanfranchi M, Malek AM. Effect of antiplatelet therapy on thromboembolism after flow diversion with the pipeline embolization device. J Neurosurg 2013;119:1603-1610

13. Szikora I, Berentei Z, Kulcsar Z, Marosfoi M, Vajda ZS, Lee W, et al. Treatment of intracranial aneurysms by functional reconstruction of the parent artery: the budapest experience with the pipeline embolization device. AJNR Am J Neuroradiol 2010;31:1139-1147

14. Puffer RC, Kallmes DF, Cloft HJ, Lanzino G. Patency of the ophthalmic artery after flow diversion treatment of paraclinoid aneurysms. J Neurosurg 2012;116:892-896

15. Leung GKK, Tsang AC, Lui WM. Pipeline embolization Device for intracranial aneurysm: a systematic review. Clin Neuroradiol 2012;22:295-303 